\title{
Automated method for buried object detecting using ground penetrating radar (GPR) survey
}

\author{
Israa J. Muhsin, Hessian Saleh Mahdi
}

Physics department, College of science, Baghdad University

E-mail: israaphsics@gmail.com

\begin{abstract}
Ground Penetrating Radar (GPR) is a nondestructive geophysical technique that uses electromagnetic waves to evaluate subsurface information. A GPR unit emits a short pulse of electromagnetic energy and is able to determine the presence or absence of a target by examining the reflected energy from that pulse. GPR is geophysical approach that use band of the radio spectrum. In this research the function of GPR has been summarized as survey different buried objects such as (Iron, Plastic(PVC), Aluminum) in specified depth about $(0.5 \mathrm{~m})$ using antenna of $250 \mathrm{MHZ}$, the response of the each object can be recognized as its shapes, this recognition have been performed using image processing such as filtering. Where different filters like (DC adjustment, triangular FIR, delete mean trace, FIR) have been applied on output image as well as the simulation of the soil and the buried objects layers have been obtained using GPR simulation program.
\end{abstract}

Key words

$G P R$, $R A D A R$, Image processing.

Article info.

Received: Nov. 2013

Accepted: Mar. 2014

Published: Apr. 2014

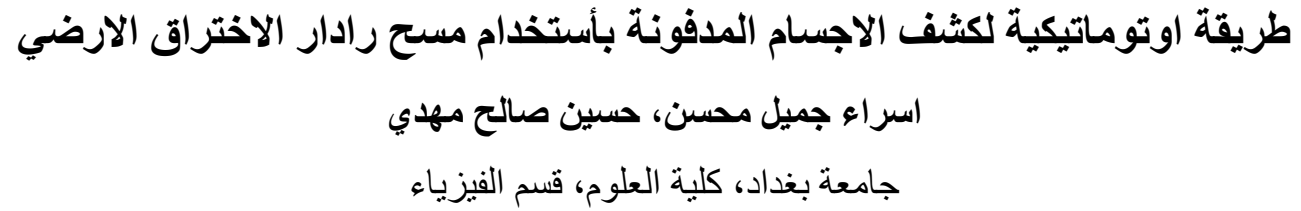

الخلاصة

رادار الاختراق الارضي هو من الاساليب الجيوفيزيائية الفعالة التي تستخدم الموجات الكهرومغناطيسية لاستخراج

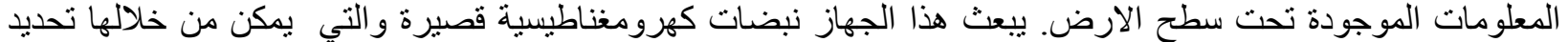

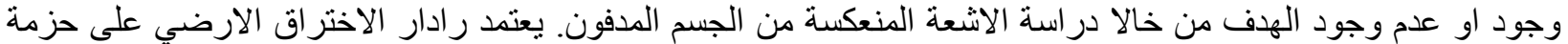

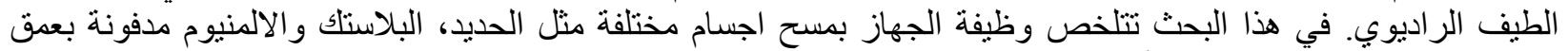

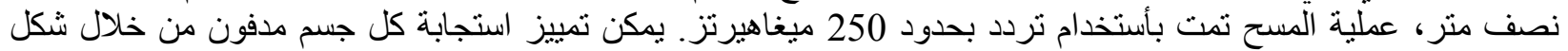

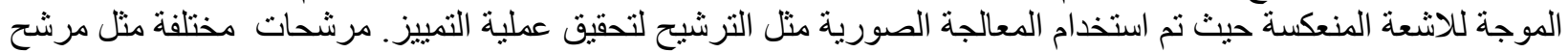

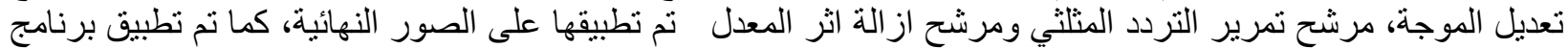
المحاكاة على طبقة الارض ترضير السطحية والطبقة التي تم دفن فيها الاجسام.

\section{Introduction}

Ground Penetrating Radar (GPR) is one of the most promising and widely used techniques for close-range detection and identification for buried targets [1-2]. GPR functions by sending electromagnetic waves into the ground and by sampling the backscattered electromagnetic echoes which are affected by the permittivity, permeability and conductivity of the material beneath the ground. By utilizing appropriate signal 
processing techniques, we can image the electrical properties of subsurface under test[3]. Therefore, GPR has the ability to detect buried objects, including both metallic and non-metallic materials. In recent years, GPR has increasingly been applied to the problems of locating pipes and cables, ice mapping, geographical surveying and landmine detection. One important consideration for developing a GPR system is the range resolution. The range resolution of a radar system is defined as "the ability to distinguish between two targets solely by the measurement of their ranges (distance from the radar); usually expressed in terms of the minimum distance by which two targets of equal strength at the same azimuth and elevation angles must be spaced to be distinguishable" [4]. The range resolution $(\Delta R$ ), i.e., the depth resolution for the GPR case, is related to the bandwidth $B$ of the system and the propagation velocity $v$ in the media. The relationship is given as [5]

$$
\Delta R=\frac{v}{2 B}
$$

When expanding the bandwidth, the propagation attenuation in the soil for the high frequency components of the wave will increase dramatically, corresponding to a sharp decrease of the penetrating depth. The result: very little back-scattered signal for high frequency waves is collected by the receiving antenna. Therefore, by increasing the bandwidth, one cannot guarantee sufficient interrogation energy[6].

RADAR technique is usually employed to detect back-scattered radiation or reflection from a buried target. Although forward scattering of the target can also be used, it is seldom employed due to the need for at least one antenna to be buried and some kind of image transformation to be applied to the measured data. All of the work discussed here is based on target backscattering measurement. Fig.1 shows the block diagram of a general subsurface radar system. The design is typical of a base-band, subsurface pulsed radar system. The subsurface radar system records microwave radiation that passes through the ground and is returned to the surface. A transmitter sends a microwave signal into the subsurface. As the radar wave propagates at a velocity that is dependent upon the dielectric constant of the subsurface medium, changes in the dielectric constant that are due to changes in the subsurface materials will cause the radar wave to reflect. The time it takes for the electromagnetic energy to return to the surface is related to the depth at which the energy was reflected. Interpretation of this reflected energy yields information on structural variation of the near subsurface or the depth of the buried objects [6].

\section{Methodology and Materials}

For this paper there is an obvious target and one that is not quite so apparent. The obvious target is the detection of the buried object, which may have dielectric constant, electrical conductivity, and magnetic characteristics different from the soil material surrounding it on the outside or the air/water contained inside. These objects constructed of plastic (PVC), Iron and Aluminum. The detection of these objects can be achieved using Ground Penetrating Radar (GPR) survey, where an electromagnetic radio energy (radar) pulse was directed into the subsurface, followed by measurement of the elapsed time taken by the signal as it traveled downward from the transmitting antenna, partially reflected off a buried feature, and was eventually returned to the surface, where it was picked up by a receiving antenna. Reflections from different depths produced a signal trace, which was a function of radar wave amplitude versus time [8]. Antenna frequency, soil moisture conditions, clay content, salinity, and the amount of Iron 


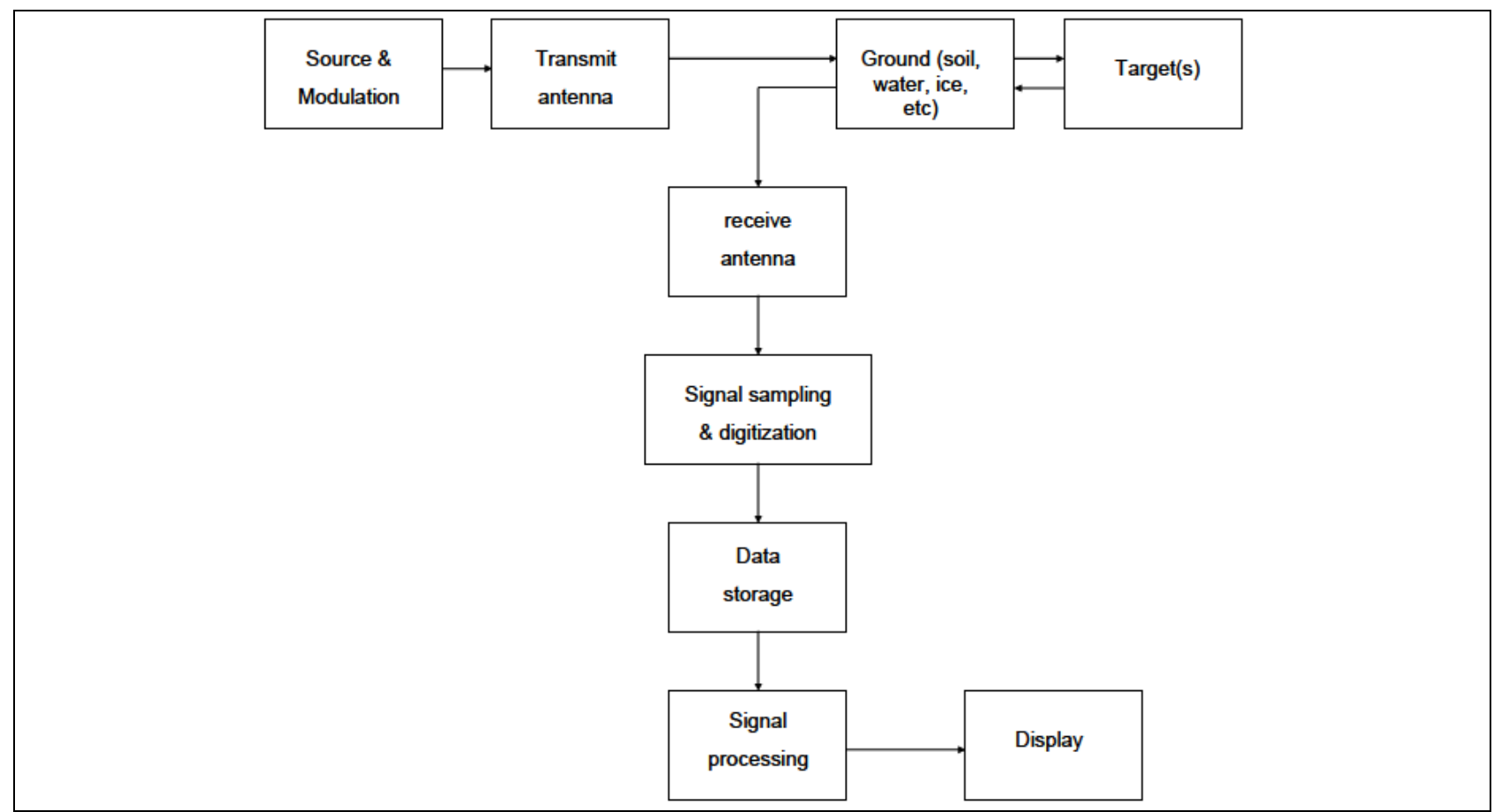

Fig.1: The block diagram showing the operation of a typical base-band subsurface RADAR system [7].

oxide present all have a substantial influence on the distance beneath the surface to which the radar signal penetrated. Differences in the dielectric constant across a discontinuity, govern the amount of reflected energy returning to the surface. The studied area for achieving this research was Baghdad university- College of Science as shown in Fig. 2 with 442253.8 E- $442294.35 \mathrm{E}$ longitude and $3681707.6 \mathrm{~N}-3681735.44 \mathrm{~N}$ latitude, UTM projection and zone $38 \mathrm{~N}$. The objects have been buried in clay (saturated) soil with 8-12 relative dielectricity and $0.1-1$ conductivity $(\mathrm{s} / \mathrm{m})$. The shielded $250 \mathrm{MHz}$ antenna is a general-purpose antenna. It is used for medium penetration depth and medium resolution. It is commonly used for utility detection, Underground Storage Tanks and void detection. The methodology of this work includes many steps as follows:

Step One: Initialize the study area by modify, and then excavated and buried objects to be disclosed, which include Plastic(PVC), Iron and Aluminum.

Step Two: Detecting buried objects by scanning using Ground Penetration Radar devise of mala type as shown in Fig. 3.

Step Three: The preparation of a radar penetration setup such as time window, velocity, point interval, antenna separation, sampling frequency as follow:

Time window $=48.1 \mathrm{~ns}$, velocity $=75 \mathrm{~m} / \mathrm{ns}$, point interval $=0.02 \mathrm{~m}, \quad$ sampling frequency $=4034.23$.

Step Four: Using image processing software such as RAD explorer, ground vision 2 for the purposes of image filtering and removing the noise. This signals may be generated from interference buried signals with soil components signals. in addition to the noise generated by soil moisture as characterized in the study area soil as agricultural soil moist. 
Step Five: Filtering is a general term for transforming image intensities in order to enhance or improve the quality of an image prior to interpretation. There is no general theory of image enhancement. The radar images are processed for visual interpretation which is a highly subjective process. The software is sufficiently flexible to allow the user to try different processes which may bring out different features in datasets. The filters used with the radar data are background removal, low pass or smoothing filters and high pass or sharpening filters. Background removal attempts to remove slowly varying background intensities by first approximating them, and then subtracting this approximation from the original image. This is done by taking a mean value for a section and subtracting an amount proportional to this from each value along this section. The smoothing filter achieves noise reduction and reduces spikes. A kernel size is specified by the user and for each point in the data the surrounding values within this kernel, including the point itself, are summed. By using ground vision2 and RAD explorer program software the arithmetic mean replaces the data point. The high pass or sharpening filter highlights fine details and enhances detail that has been blurred during image acquisition. Each data point is replaced by an amount proportional to the difference between the point and the mean of its neighbors [9]. Low contrast images can be improved by contrast stretching which increases the dynamic range of the grey levels in the image being processed. In this paper many filter have been applied on the output image such as (DC adjustment, delete mean trace, FIR, triangle FIR) for enhancement purpose.

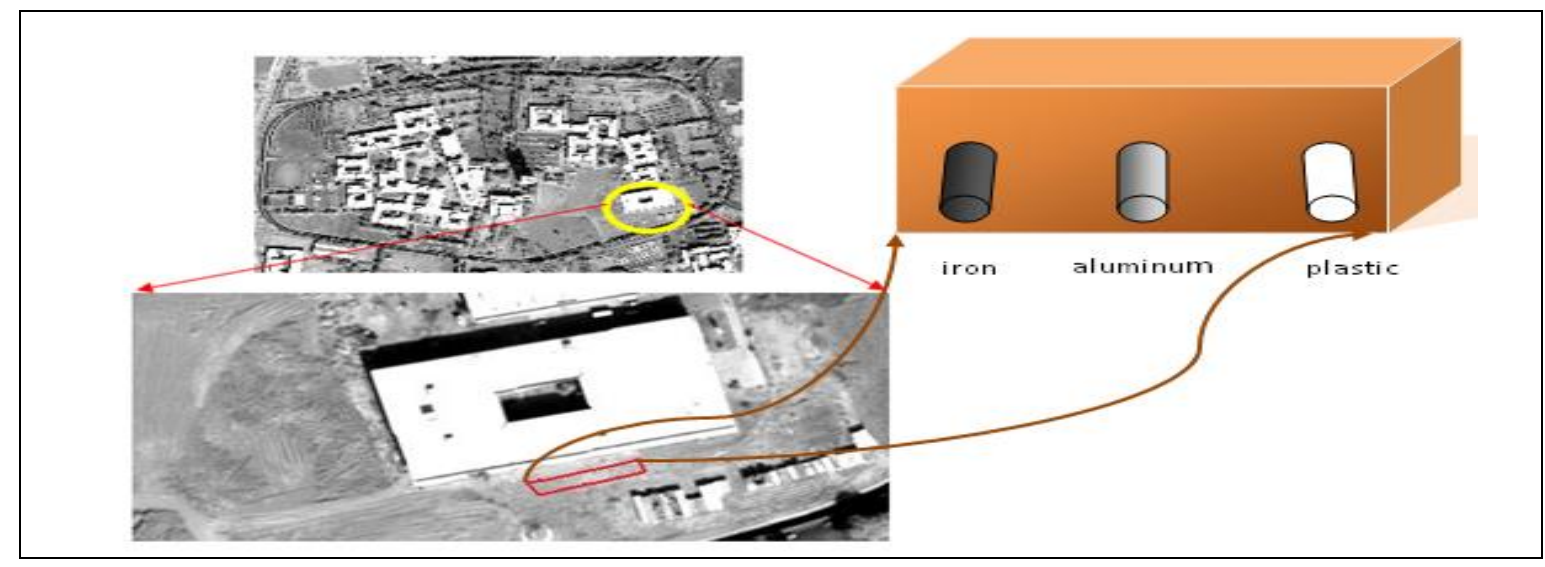

Fig.2: The studied area .

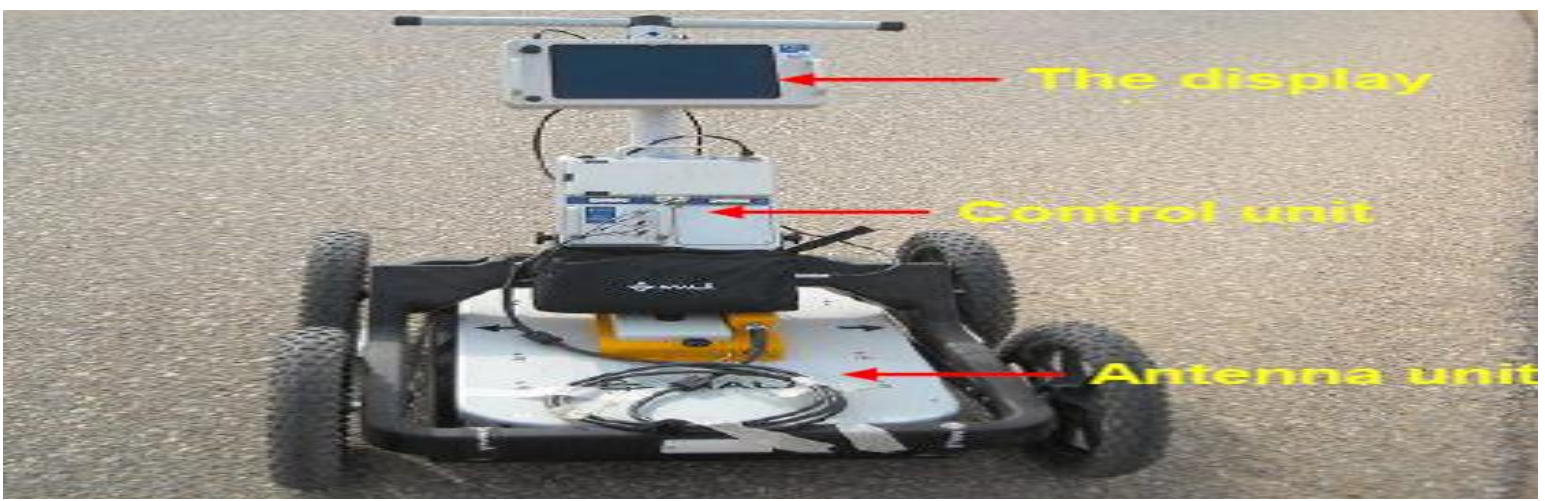

Fig.3: The GPR Device type (mala). 


\section{Simulation Process}

The general algorithm for simulation includes:

- Calculation of the amplitude of a signal reflected from each layer of a multi-layered media.

- Calculation of the two-way time delay of a signal reflected from each layer.

- Simulation of echo signals reflected from the multi-layered media, clutters, impulse noise and speckle noise.

- Construction of the synthetic range profile. It is done for each of $N$ positions of the transmitter-receiver system. At this stage, the matrix of $M$ columns is simulated. Each column of this matrix contains the echo signal simulated for one of $M$ transmissions. This signal matrix is used for construction of a synthetic range profile by the steppedfrequency approach.

\section{Results and Discussion}

The proposed technique employs a combination of detection, filtering for clutter removing and image processing to provide a high-resolution image of the sub-surface in near real-time facilitating straightforward data interpretation and providing accurate depth and azimuth location information. The process of detecting buried objects, which include Plastic (PVC), Aluminum and Iron carried out using Ground Penetration Radar as shown in Fig.4. where the form of radar waves reflected from the buried material and received by the scanning device can be notice. Each subject reflects the wave in way different from other materials, based on the dielectric constant for each subject. These waves received not be cleared and well due to overlap between them and the reflected waves from the surrounding soil. In addition to the reflected waves from the elements and oxides that make up the soil. As the soil moisture also affects the data received, where the soil that has characterized the study as saturated soil.
So, the data of GPR must be filtered and analysis, the analysis of this data carried out by processing it using different filtering techniques and gains. Filtering is the use of mathematical processing algorithms to clean noise from the data and/or enhance certain characteristics of the data. Gain is a value by which raw data are multiplied to enhance low-amplitude reflections. Signal amplitude commonly decreases exponentially at increasing travel. This was compensated by designing a custom time gain that increases the signal strength. The result of applying (Dc Adjustment, delete mean trace, FIR, triangle FIR) filters on the images can be shown in the Fig. 5and 6 respectively. The obtained results show that the buried iron component has shown a stronger signal than the rest of the buried material due to the chemical and physical properties of elemental iron with a dielectric constant 14.3 and electrical conductivity $1 \times 10^{7} \mathrm{~s} / \mathrm{m}$.

The obtained results in this research show that the application of the (Delete mean trace) filter has clear image more than the rest of the filters due to the possibility of this filter to noise removing from the overlapping signals of the original soil components, addition to moisture and other impurities buried with objects.

This does not mean that the rest of the filters is not important, but on the contrary, all of the filters have been applied in this research are useful and have given good results to improve the resulting image of the survey, which was by a Ground Penetration Radar. In the other hand simulation of soil layers and materials buried have been achieved, take into consideration the existence of four layers of dry soil, moist soil, the type of objects buried, and then a layer of soil beneath the element of this simulation was made using the program (GPR simulation) and the obtained results are shown in Fig. 7. 


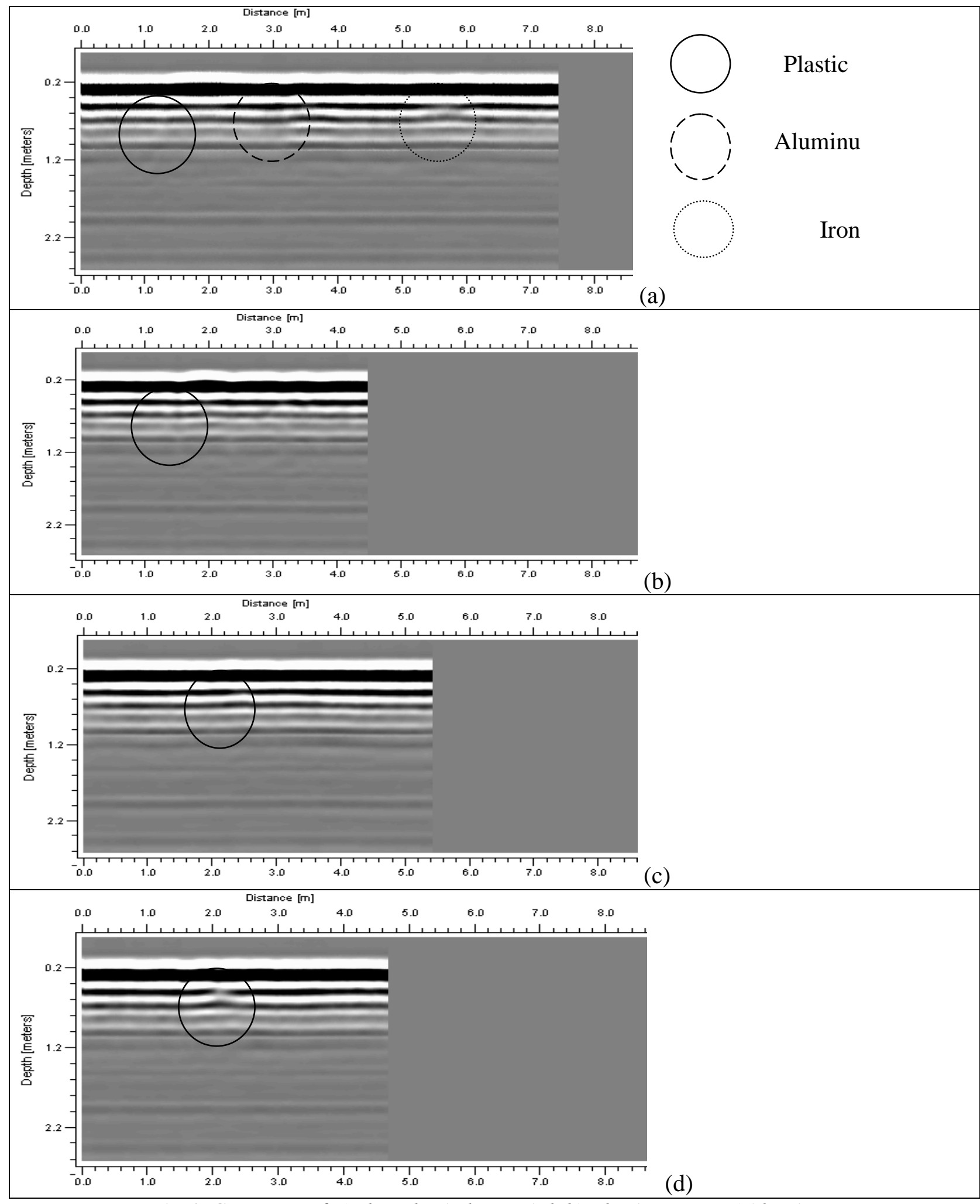

Fig.4: GPR scan of a)Three buried material, b) Plastic matter, c)Aluminum matter, d) Iron matter. 


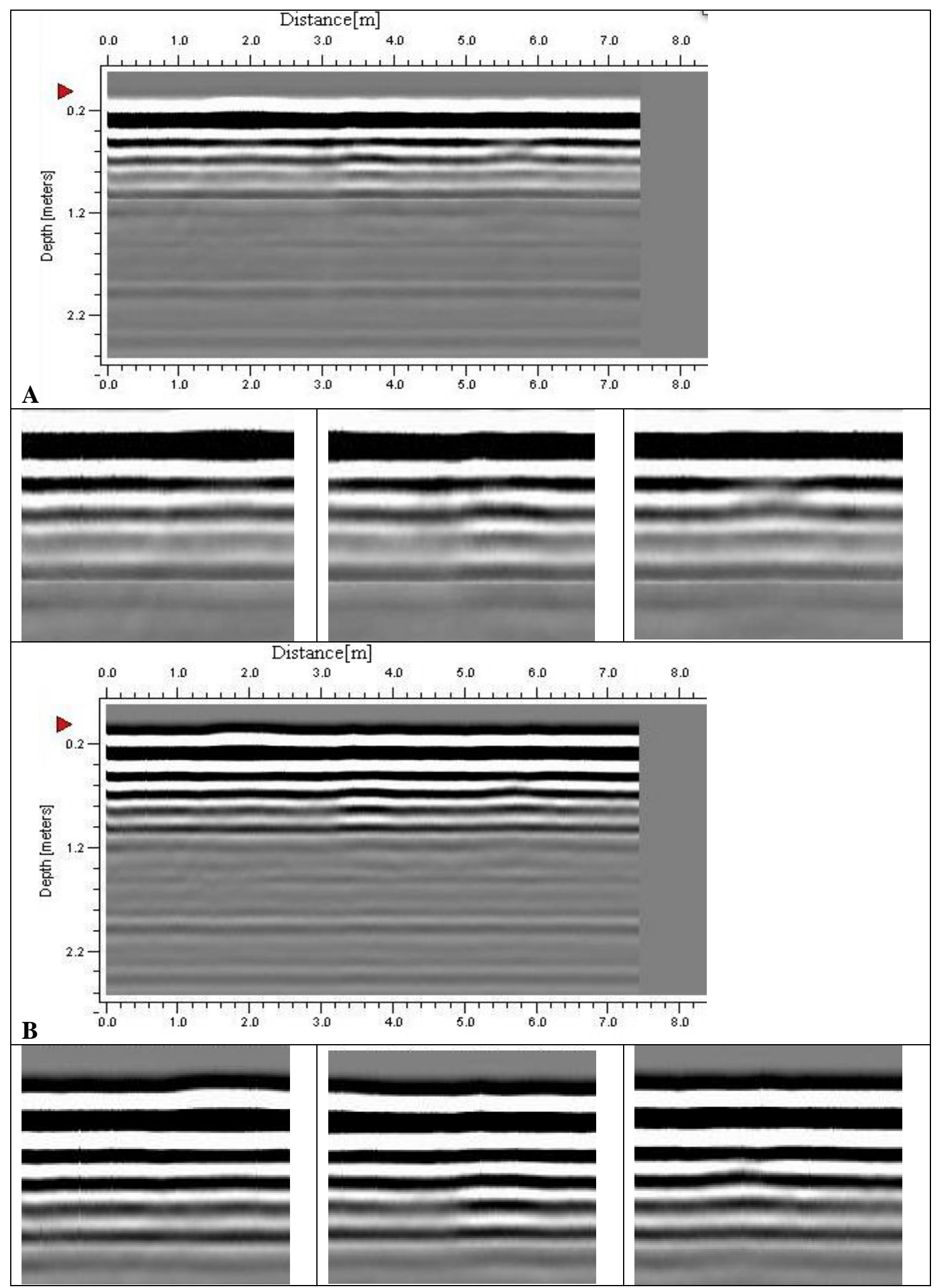

Fig.5: The filtered image with zooming of each buried objects A) apply dc adjustment filter. B) Apply FIR filter. 


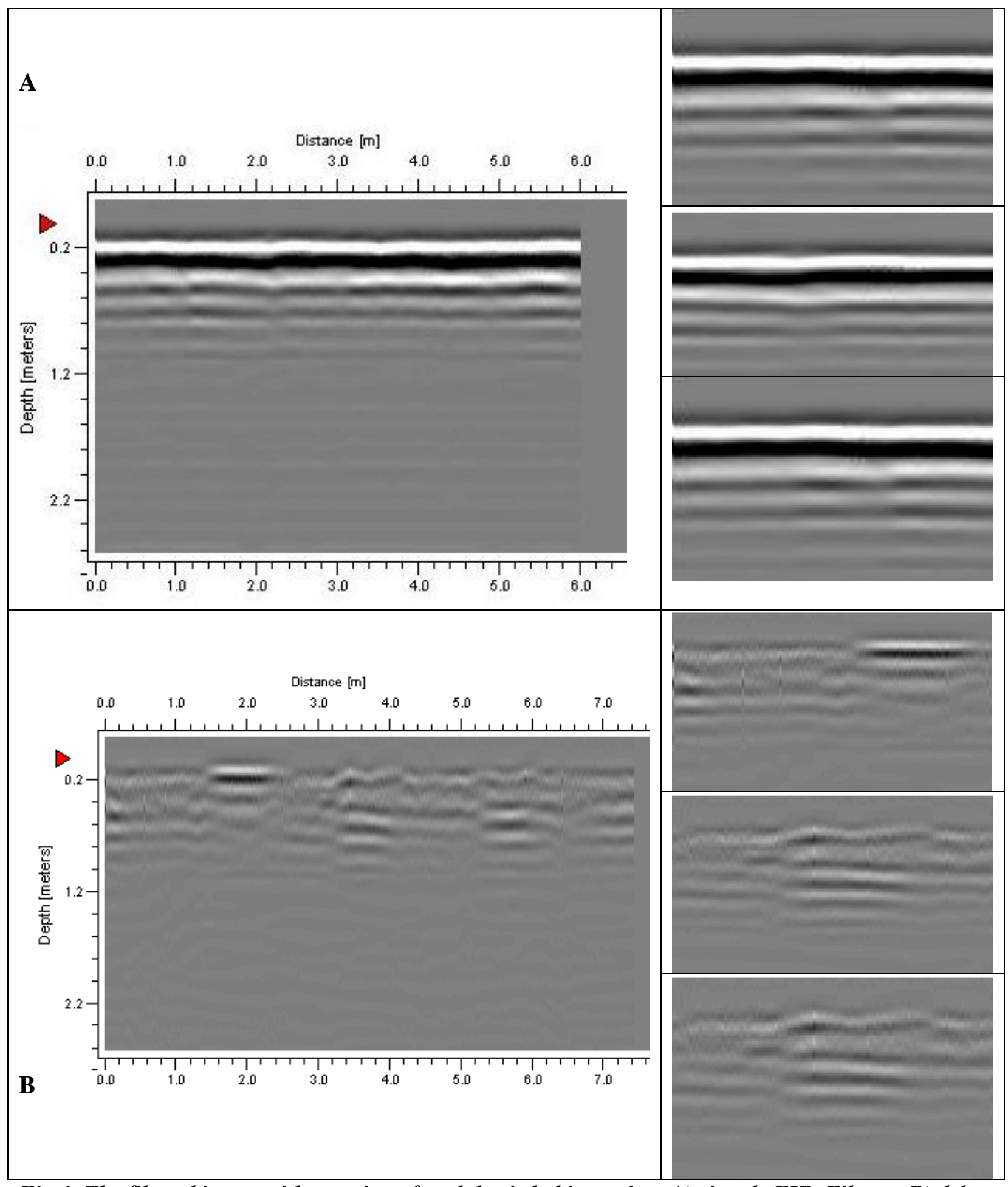

Fig.6: The filtered image with zooming of each buried object using A)triangle FIR. Filter, B) delete mean trace. 


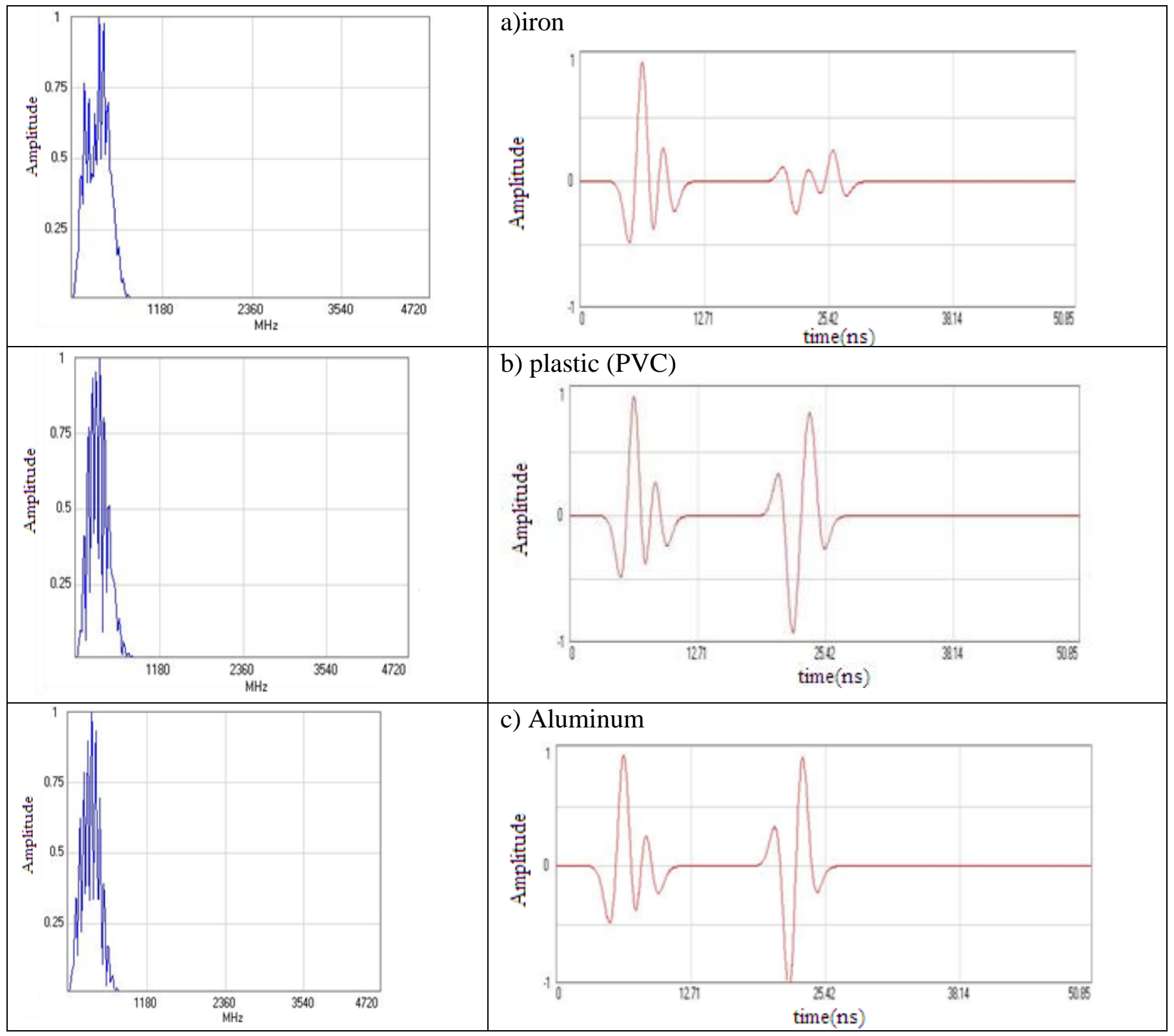

Fig.7: The simulation of a)Iron, b) plastic (PVC), c)Aluminum .

\section{Conclusions}

1. During the GPR surveying it's important that the fields be as dry as it could be because of the distortion effect of the water to the reflected part of the radio wave that transmitted from the GPR antenna which forms the output image.

2. In general, after data acquisition and processing using ground vision2 software and implementing different filters, it was found that the delete mean trace filter consider being the best one.
3. from the output images of the buried materials such as iron, Aluminum, and plastic its apparently that iron and Aluminum elements have approximately the same shape and there are differ from the plastic element, these belong to the difference of the dielectric and other physical and chemical characteristic. 


\section{References}

[1] B.,Scheers, Ultra-Wideband, Ph.D Thesis, University of Catholique, Louvainla-Neuve, Belgium, (2001).

[2] D. J. Daniels, "Ground Penetrating Radar", Encyclopedia of RF and icrowave Engineering, vol.2, John Wiley \& Sons, Inc., Hoboken, New Jersey, United States, (2005) 1833-1846.

[3] C. Soto, E. Rogelio, M.Sc. Thesis, University of Manitoba, University of Manitoba, Department of Electrical and Computer Engineering, Canada, (2003).

[4] IEEE Standard Radar Definitions, IEEE Std. 686-1990, p. 25.

[5] M. Soumekh, Synthetic Aperture Radar Processing with MATLAB Algorithms, John Wiley and Sons Inc., New York, United States, (2002).
[6] L., Teck Yong, M.Sc. thesis, department of Electrical Enginnering, California December, (2003).

[7] D. J. Daniel "Ground Penetrating Radar" $2^{\text {nd }}$ edition, published by: the institution of electrical engineers, london, united kingdom, (2004).

[8] B. J. Allred, N. R. Fausey, L. Peters, Jr., C. Chen, J. J. Daniels, H. Youn., Applied Engineering in Agriculture American Society of Agricultural Engineers, 20, 3(2004) 307-318,.

[9] Vanessa S. Blake, Computer applications and quantitative methods in archaeology 1994 Edited by Jeremy Huggett and nick Ryan. Tempus reparatum. BAR international series 600, pp (175-180). 